\title{
The Synthesis and Characterization of Cobalt-Rice Husk Silica Nanoparticles
}

\author{
Farook Adam ${ }^{1, *}$, Jeyashelly Andas ${ }^{1}$ and Ismail Ab. Rahman ${ }^{2}$ \\ ${ }^{1}$ School of Chemical Sciences, Universiti Sains Malaysia, 11800 Penang, Malaysia \\ ${ }^{2}$ School of Dental Sciences, Health Campus, Universiti Sains Malaysia, 16150 Kubang Kerian, Kelantan, Malaysia
}

\begin{abstract}
Spherical and needle-like cobalt nanoparticles incorporated on rice husk silica was synthesized by a simple, rapid and eco-friendly sol-gel route at room temperature. This was achieved by direct mixing of $20 \mathrm{wt}^{\%} \mathrm{Co}^{2+}$ with sodium silicate from rice husk under ambient conditions to form RH-20Co. The cobalt was found to be isomorphously substituted in the silica matrix. A good dispersion of cobalt nanoparticles was evidenced from X-ray diffraction. Transmission electron microscopy confirmed the formation of well-dispersed and spherical cobalt nanoparticles with an average particle size of $5.6 \mathrm{~nm}$. The magnetic properties of this material were measured with vibrating magnetometer. The saturation magnetization, $M_{s}$ and coercive force, $H_{c}$ was found to be $0.245 \mathrm{emu} / \mathrm{g}$ and 304.09 Oe respectively.
\end{abstract}

Keywords: Cobalt nanoparticles, spherical, rice husk silica, sol-gel, magnetization.

\section{INTRODUCTION}

Nanoparticles have been regarded as an exceptional and important material due to its well-established properties such as size and surface effects $[1,2]$. These materials are applicable in miscellaneous fields such as catalysis, electronics, optical, biotechnology and magnetism [3-5]. Among known transition metals, cobalt undoubtedly has gained wide interest in numerous catalytic studies. For example, cobalt nanoparticles supported on silica in steam reforming ethanol [6], $\mathrm{CoFe}_{2} \mathrm{O}_{4}$ and $\mathrm{Co}_{3} \mathrm{O}_{4}$ nanocrystals as heterogeneous catalyst for cyclohexane oxidation [7] and also methane combustion [8]. Owing to its broad applications, various methods have been employed by researchers to produce unique Co or Co bimetallic nanoparticles which includes thermal decomposition [9, 10], pulsed laser irradiation [11], chemical vapor condensation (CVD) [12], co-precipitation [13], reversed micelle technique [14], reduction of cobalt chloride [15] and thermolysis [16]. Most recently, a ferromagnetic cobalt nanoparticle between $35-200 \mathrm{~nm}$ size range was successfully synthesized by a modified polyol technique [4]. Additionally, cobalt nanoparticles were synthesized by reverse microemulsion consisting of either neutral or ionic surfactants [17]. Furthermore, Fu and co-workers [18] have developed spherical core cobalt/silica nanoparticles by activated plasma evaporation of the metal. However, most of these existing procedures are expensive, complicated, time consuming and involves organic solvents. For instance, thermal decomposition methodology requires long period of calcination and high temperature as reported by Yang et al., [10].

Silica is an inert support material with a high surface area $[19,20]$. It has been widely used in ceramics and electronics

*Address correspondence to this author at the School of Chemical Sciences, Universiti Sains Malaysia, 11800 Penang, Malaysia; Tel: +60 4 6533567; Fax: +60 4 6574854; E-mail: farook@usm.my, farook_dr@yahoo.com
[21]. Rice husk, an abundantly available agricultural waste has emerged as an ideal source of high grade amorphous silica. Many studies have been reported on the use of silica from rice husk as a catalyst support [22-25]. Sol-gel process is claimed to be a simple and effective synthesis pathway for the production of nanomagnetite [26], silica nanotubes [27] and nanocomposites [28, 29]. Moreover, recently, Zaky et al., [30] obtained a narrow size distribution of silica nanoparticles with an average size of $50-70 \mathrm{~nm}$ from semiburned rice straw ash. Thus, herein, for the first time, we propose a simple and novel sol-gel route to synthesize cobalt nanoparticles supported on rice husk silica with unique morphology and magnetic properties.

\section{MATERIALS AND METHODS}

Rice husk ( $\mathrm{RH})$ was obtained from a local rice milling plant in Penang, Malaysia. Cobalt nitrate $\left(\mathrm{Co}\left(\mathrm{NO}_{3}\right)_{2} \cdot 6 \mathrm{H}_{2} \mathrm{O}\right)$, R\&M Chemicals, 99\%) was used as the cobalt precursor. Sodium hydroxide (QRec, 99\%) and nitric acid (QRec, 69\%) were of analytical grade and used without further purification.

Washing and pretreatment of RH was carried out according to the procedure reported by Adam et al., [24]. Washed and dried RH $(30.0 \mathrm{~g})$ was stirred in $500 \mathrm{~mL}$ of $1 \mathrm{M} \mathrm{HNO}_{3}$ overnight, to enable the removal of metals from the RH [25]. This acid treated RH was washed thoroughly with copious amount of distilled water to ensure the absence of $\mathrm{NO}_{3}{ }^{-}$and subsequently dried in an oven at $110^{\circ} \mathrm{C}$ for $24 \mathrm{~h}$. The resulting sample was denoted as $\mathrm{RH}-\mathrm{HNO}_{3}$. The weighed $\mathrm{RH}-$ $\mathrm{HNO}_{3}$ was added to $500 \mathrm{~mL}$ of $1 \mathrm{M} \mathrm{NaOH}$, stirred vigorously for $24 \mathrm{~h}$, suction filtered to obtain the residue, $\mathrm{RH}$ $\mathrm{NaOH}$ which was oven dried at $110^{\circ} \mathrm{C}$ for one day. $\mathrm{RH}-$ $\mathrm{NaOH}$ was weighed and recorded. The amount of silica extracted from $\mathrm{RH}$ was assumed to be the difference in the mass of $\mathrm{RH}-\mathrm{HNO}_{3}$ and $\mathrm{RH}-\mathrm{NaOH}$ [25]. The filtered sodium 
silicate solution was kept in a covered container to be used as the source of silica. Preparation of RH-Silica was done following the published literature [22] but the $\mathrm{pH}$ was maintained at 3. RH-Silica was synthesized by titrating the sodium silicate with 3.0 $\mathrm{M} \mathrm{HNO}_{3}$ until it reached $\mathrm{pH} 3$. A yellowish-brown suspension was observed during the titration. The observed suspension was aged for 2 days in a covered container. The gel was recovered by centrifugation and suction filtered, washed with copious amount of distilled water and oven dried at $110^{\circ} \mathrm{C}$. The dried catalyst was ground to yield $3.30 \mathrm{~g}$ of RH-Silica.

Cobalt - rice husk silica was prepared by titrating the sodium silicate with $130 \mathrm{~mL}$ of $3 \mathrm{M} \mathrm{HNO}_{3}$ containing 20 wt.\% of $\mathrm{Co}\left(\mathrm{NO}_{3}\right)_{2} \cdot 6 \mathrm{H}_{2} \mathrm{O}$ to obtain $\mathrm{RH}-20 \mathrm{Co}$. Briefly, the titration was carried out until pH 3 and the suspension obtained was aged in a covered container at room temperature for $48 \mathrm{~h}$. After 2 days of ageing, the resulting gel was washed with distilled water, centrifuged at $4000 \mathrm{rpm}$ and finally recovered by suction filtration. The obtained solid material was subsequently oven dried at $110^{\circ} \mathrm{C}$ for $18 \mathrm{~h}$ and finely hand-ground to yield $3.25 \mathrm{~g}$ of $\mathrm{RH}-20 \mathrm{Co}$.

The synthesized material was characterized by fourier transformed infrared measurements (FT-IR, Perkin Elmer System 2000), ${ }^{29} \mathrm{Si}$ MAS nuclear magnetic resonance technique $\left({ }^{29} \mathrm{Si}\right.$ MAS NMR, Bruker DSX-3000), $\mathrm{N}_{2}$ sorption analysis (Micromeritics Instrument Corporation model ASAP 2000, Norcross) and X-ray diffractometry (XRD, Kristallofex Siemens, model D5000) equipped with $\mathrm{Cu} \mathrm{K}$ radiation $(\lambda=1.50 \AA)$. The cobalt content was determined by atomic absorption spectroscopy (AAS, Perkin Elmer, model 3100). Prior to AAS analysis, the sample was digested with aqua regia and hydrofluoric acid solution. Material morphology was recorded on a transmission electron microscope (TEM, model Phillips CM12). Particle size measurements were conducted with Analysis Image Processing Cell A software. Mapping analysis was obtained by means of scanning electron microscope (SEM, model 50VP) equipped with Oxford Inca 400 Energy Dispersive X-ray (EDX). The mor- phology and roughness of the surface was determined by atomic force microscopy (AFM, ULTRAObjective) employing a non-contact mode. Magnetic property of the nanoparticles was investigated by vibrating sample magnetometer (VSM, DMS, model 8810) at $300 \mathrm{~K}$, in the applied field sweeping from -10 to $10 \mathrm{kOe}$. The amount of sample used was $0.018 \mathrm{~g}$.

\section{RESULTS AND DISCUSSION}

\subsection{Material Characterization}

Fig. (1) illustrates the FT-IR spectra of RH-Silica and RH-20Co. The observed bands are similar to the work published elsewhere [31, 32]. The band at $967 \mathrm{~cm}^{-1}$ in RH-Silica disappeared after introducing cobalt into the silica framework. This occurrence can be due to the presence of cobalt silicate or hydrosilicate [33]. Reaction between small cobalt particles with hydroxyl groups present on silica surface can form scarcely reducible cobalt silicate [14]. The inset spectrum in Fig. (1) clearly demonstrates a band at $660 \mathrm{~cm}^{-1}$ for $\mathrm{RH}-20 \mathrm{Co}$ which is absent in RH-Silica. This band is ascribed to spinel type $\mathrm{Co}-\mathrm{O}$ bond corresponding to $\mathrm{Co}_{3} \mathrm{O}_{4}[34,35]$. It confirms the $\mathrm{M}-\mathrm{O}$ stretching vibration in which $\mathrm{Co}^{2+}$ is tetrahedrally coordinated [36]. The presence of $\mathrm{Co}_{3} \mathrm{O}_{4}$ and $\mathrm{CoO}$ can be further evidenced from XRD results. This is in agreement with zirconia promoted silica supported cobalt catalyst subjected to air drying at $100^{\circ} \mathrm{C}$ which showed decomposition of cobalt nitrate into intermediate cobalt silicate phase and finally into $\mathrm{Co}_{3} \mathrm{O}_{4}$ [37].

The ${ }^{29} \mathrm{Si}$ MAS NMR spectra of the materials are shown in Fig. (2). RH-Silica exhibit $\mathrm{Q}^{3}$ and $\mathrm{Q}^{4}$ signals at 101.4 and $110.7 \mathrm{ppm}$. The $\mathrm{Q}^{3}$ and $\mathrm{Q}^{4}$ silicon centres are attributed to the $(\mathrm{Si}-\mathrm{O}-)_{3} \mathrm{Si}(\mathrm{OH})$ and $(\mathrm{Si}-\mathrm{O})_{4} \mathrm{Si}$ groups respectively [38]. However, RH-20Co shows only an upward shift at -113.2 ppm, which corresponds to $\mathrm{Q}^{4}$ signal with much broadening. Such broadening strongly suggests the interaction of silica with the paramagnetic cobalt which suggests the formation of Si-O-Co bond [39]. However, formation of cobalt nanoparticles (evidenced later) would have inhibited the

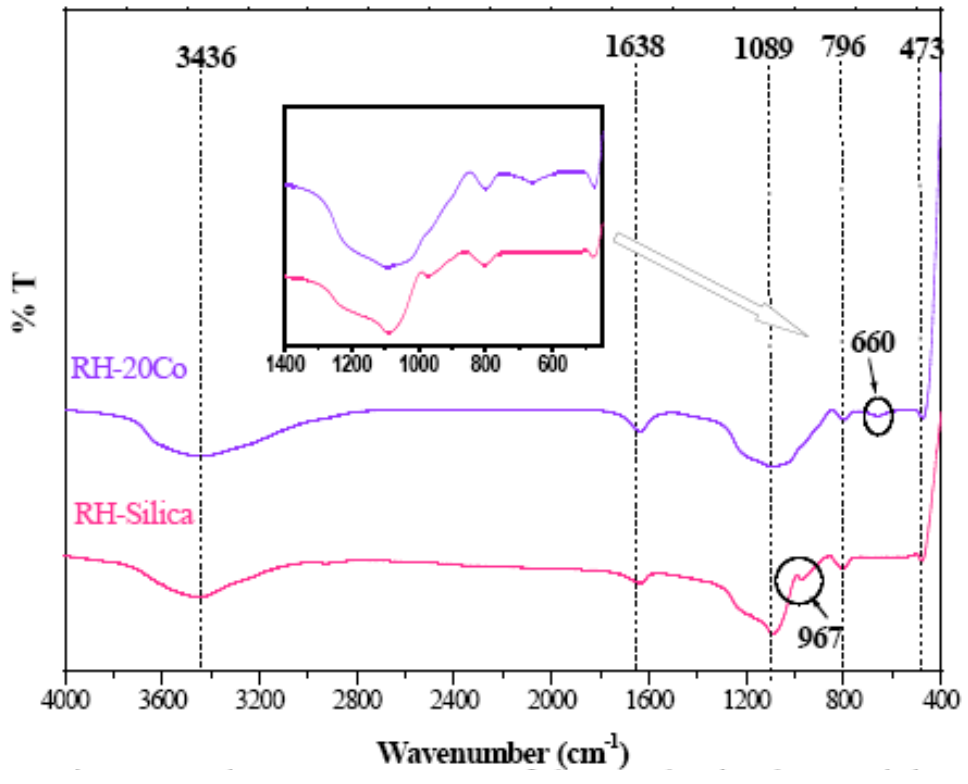

Fig. (1). The FT-IR spectra of RH-Silica and RH-20Co. 


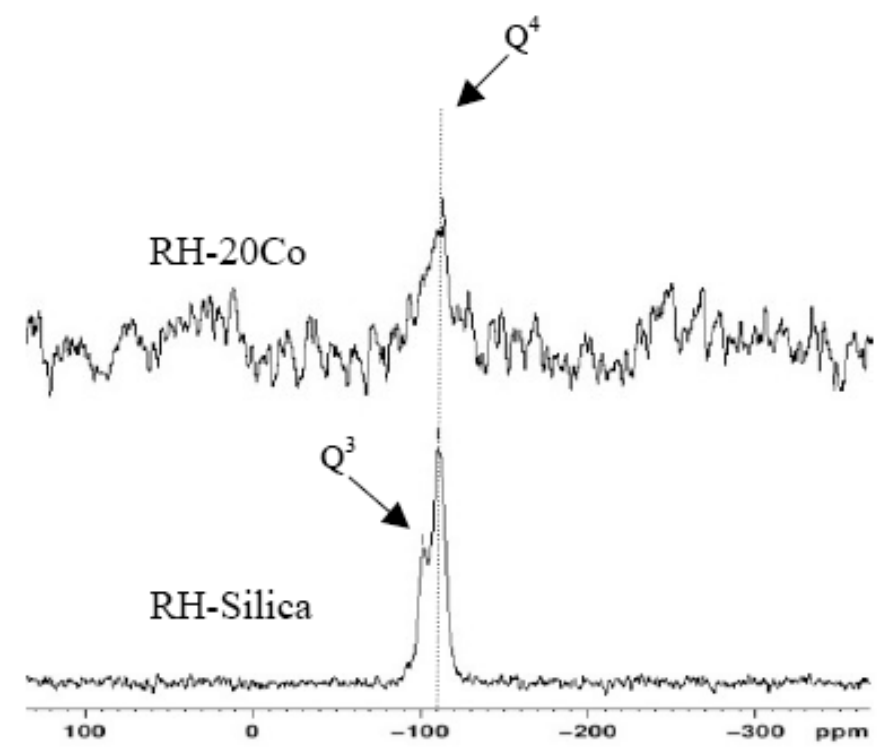

Fig. (2). The ${ }^{29}$ Si MAS NMR spectra of RH-Silica and RH-20Co.

Table 1. The Textural Properties and Summary of Data Obtained from BJH and AAS Analysis

\begin{tabular}{|c|c|c|c|c|}
\hline Catalyst & $\begin{array}{c}\mathrm{S}_{\mathrm{BET}} \\
\left(\mathrm{m}^{2} / \mathrm{g}\right)\end{array}$ & $\begin{array}{c}\text { Average } \\
\text { Pore Diameter (nm) }\end{array}$ & $\begin{array}{c}\text { Average } \\
\text { Pore Volume (cc/g) }\end{array}$ & $\begin{array}{c}{ }^{\mathrm{a}} \text { Cobalt } \\
\text { Loading (wt. \%) }\end{array}$ \\
\hline \hline RH-Silica & 312 & 3.0 & 0.27 & - \\
\hline RH-20Co & 415 & 9.0 & 0.89 & 12.3 \\
\hline
\end{tabular}

${ }^{\mathrm{a}}$ Sample preparation via microwave-assisted acid digested system.

formation of Si-O-Co which may lead to the absence of $\mathrm{Q}^{3}$ signal for RH-20Co.

The BET surface area $\left(\mathrm{S}_{\mathrm{BET}}\right)$, pore volume and pore diameter obtained from $\mathrm{N}_{2^{-}}$sorption and $\mathrm{BJH}$ analysis is shown in Table 1. Insertion of cobalt into the silica framework increased the $\mathrm{S}_{\mathrm{BET}}$ of RH-20Co ca. 33\% in comparison to that of RH-Silica. It indicates a good dispersion of cobalt in the silica matrix [40]. This is in accordance with the XRD results (evidenced later). The pore diameter along with the pore volume of RH-20Co was also increased. Such characteristics can be attributed to the incorporation of larger cations such as $\mathrm{Co}^{2+}$ in a tetrahedral geometry of $\mathrm{Si}^{4+}$. It is indeed a strong indication that cobalt has been successfully incorporated into the silica matrix. Shi and Shen [41] found that Co-MCM-41 (5-20\% Co) catalysts exhibited a similar increasing trend in pore diameter compared to that of $\mathrm{Co}-$ MCM-41. Enhancement in pore volume, pore diameter and surface area of RH-20Co may have favored the deposition of cobalt oxide species on the silica surface as suggested by $\mathrm{Li}$ and co-workers [42]. Chemical analysis by AAS for RH20 Co (Table 1) shows that $c a$. $12 \%$ of cobalt was successfully incorporated.

The $\mathrm{N}_{2}$ adsorption-desorption isotherms together with the pore size distribution patterns of both materials are depicted in Fig. (3). Adsorption isotherms of RH-Silica and RH-20Co are of Type IV, indicative of mesoporous solids [43, 44]. However, RH-20Co exhibits a H1 hysteresis loop and RHSilica shows $\mathrm{H} 2$ hysteresis loop suggesting a change in the pore structure with incorporation of cobalt. In addition, RHSilica shows narrow and monomodal pore size distribution (PSD) with average diameter of $3.0 \mathrm{~nm}$ whereas $\mathrm{RH}-20 \mathrm{Co}$ exhibits a wider range of PSD with average pore diameter of $9.0 \mathrm{~nm}$ (Fig. 3b).

Powder XRD pattern of the materials are presented in Fig. (4). A broad peak at $2 \theta=23^{\circ}$, shows the typical attributes of amorphous silica [45]. The profile of $\mathrm{RH}-20 \mathrm{Co}$ only shows weak and rather broad signals. Characteristic peaks of $\mathrm{CoO}, \mathrm{Co}_{3}(\mathrm{OH})_{4} \mathrm{Si}_{2} \mathrm{O}_{5}$ and $\mathrm{Co}_{3} \mathrm{O}_{4}$ can be identified as indicated in the pattern. This is in agreement with FTIR results discussed earlier (Fig. 1). A similar phenomenon was reported by Dutta et al., [46] in the preparation of $10 \%$ $\mathrm{Co} /$ aerogel nanoneedles. Formation of $\mathrm{CoO}$ was most likely due to the reduction of $\mathrm{Co}_{3} \mathrm{O}_{4}$. Reflections corresponding to Co could not be observed which could be due to the overlap with other signals or broadening of the XRD peak. It could also be due to the presence of very small Co particles [47]. Smaller Co particles undetected in the XRD may also lead to untraceable crystalline peaks [48].

Selected TEM micrographs of RH-Silica and RH-20Co at different magnifications are presented in Fig. (5). Figs. (5a) and (b) demonstrate amorphous porous structure of RHSilica. Highly-dispersed and spherical cobalt nanoparticles and needle-like nanoparticles can be seen in Figs. (5c) and (d) respectively. It is comparable with TEM results obtained for Co disulfide nanoparticles prepared by the reaction of 

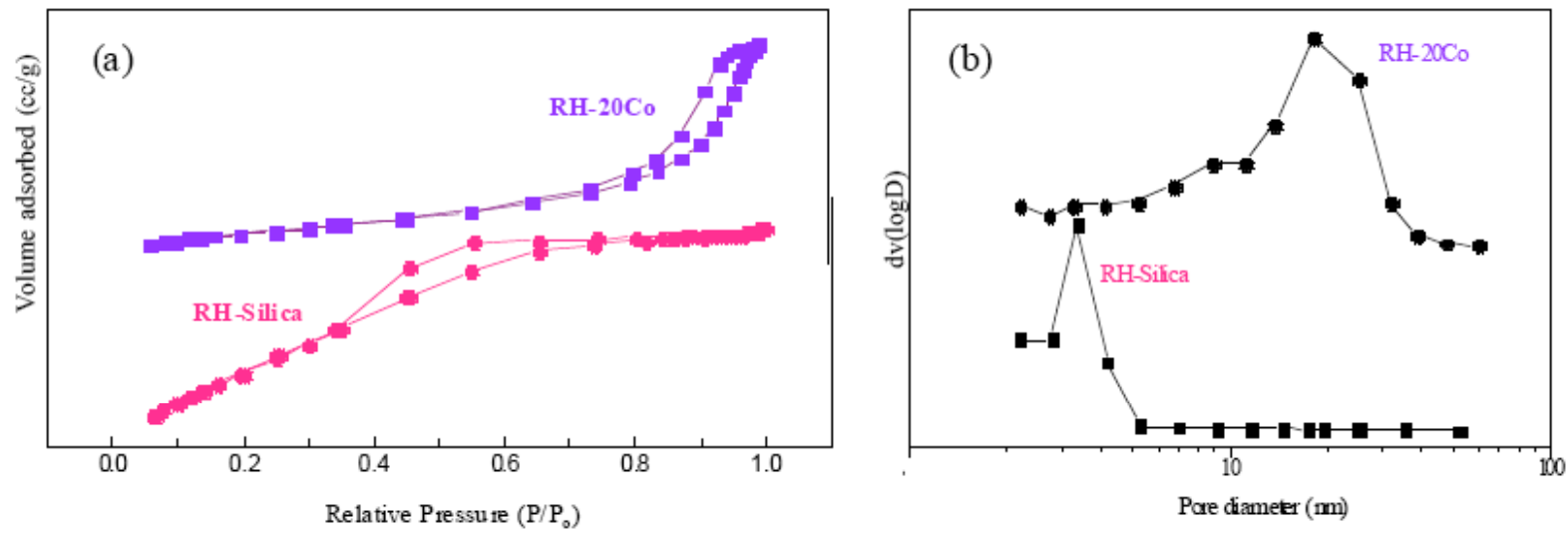

Fig. (3). The $\mathrm{N}_{2}$ adsorption-desorption analysis of (a) the isotherms of RH-Silica and RH-20Co and (b) the pore size distribution of RH-Silica and RH-20Co.

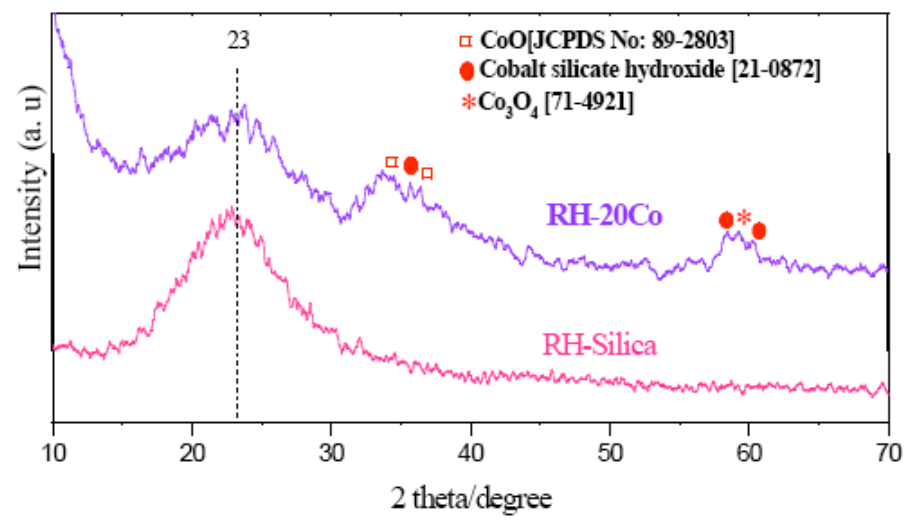

Fig. (4). The XRD patterns of RH-Silica and RH-20Co.

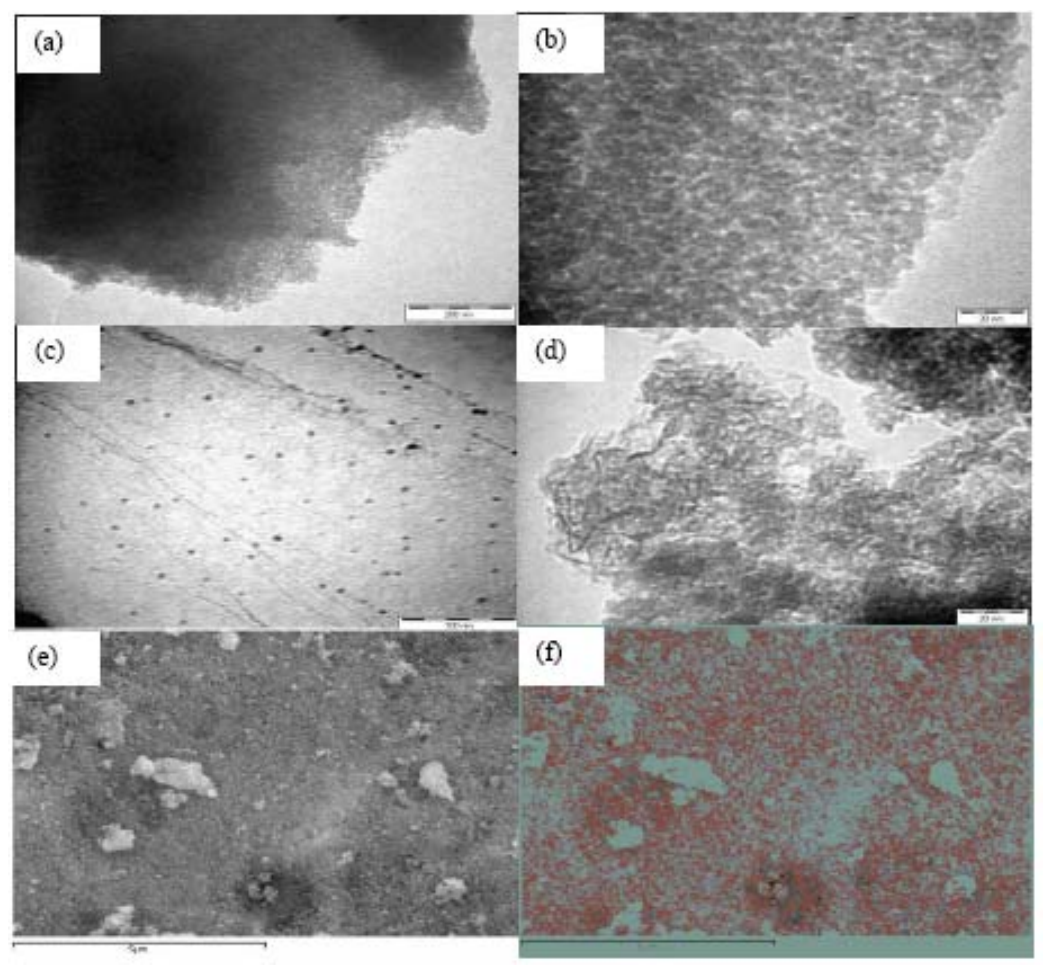

Fig. (5). Representative TEM images of (a,b) RH-Silica and (c,d) RH-20Co at different magnifications and (e,f) SEM image and cobalt mapping analysis of RH-20Co. 
$\mathrm{CoCl}_{2} \cdot 6 \mathrm{H}_{2} \mathrm{O}$ with thiourea and hydrazine in aqueous solution at a refluxing temperature of $90-95^{\circ} \mathrm{C}$ [49]. In $\mathrm{RH}-20 \mathrm{Co}$ synthesized at $\mathrm{pH} \mathrm{3}$, cobalt ions were fairly well dispersed without much agglomeration. This is presumably due to the $\mathrm{pH}$ range of 2-5 which favored adsorption of cobalt ions on negatively charged silica surface and thereby improve the cobalt dispersion [50]. Good dispersion of cobalt in RH-20Co could be further evidenced by SEM and cobalt mapping images as shown in Figs. (5e) and (f) respectively.

A narrow and uniform particle size distribution of RH20Co with a mean diameter of $5.6 \mathrm{~nm}$ is shown in the histogram (Fig. 6). All the particles were observed as monomodal without formation of large particles. This shows that the simple sol-gel method used in this study is competent in producing cobalt particles with an average size of $5.6 \mathrm{~nm}$. This is comparable to published methodology such as incipient wetness impregnation, drying and calcination in $\mathrm{NO} / \mathrm{He}$ which produced 18 wt. $\% \mathrm{Co} / \mathrm{SiO}_{2}$ nanoparticles of $\pm 4.6 \mathrm{~nm}$ diameter [51].

AFM images of RH-20Co are displayed in Fig. (7). The surface topography of the material consists of spherical shaped pore system which is in line with TEM analysis. The surface roughness mean square (rms) of RH-20Co was found to be $107.24 \mathrm{~nm}$.

\subsection{Magnetic Properties}

The magnetic hysteresis curve obtained at $300 \mathrm{~K}$ for RH20Co is depicted in Fig. (8). The corresponding saturation magnetization $\left(M_{s}\right)$, coercivity $\left(H_{c}\right)$ and remanent magnetization $\left(M_{r}\right)$ were noted to be $0.245 \mathrm{emu} / \mathrm{g}, 340.09$ Oe and $0.0115 \mathrm{emu} / \mathrm{g}$ respectively. $M_{s}$ for RH-20Co was much lower compared to $M_{b u l k C o}=166 \mathrm{emu} / \mathrm{g}$ [36]. Decrease in $M_{S}$ is mostly due to the smaller cobalt rice husk silica nanoparticles synthesized in this study. Hard magnet behavior is shown from the hysteresis loop. Materials with large $H_{c}$ (>100 Oe) are known as hard magnets [52]. The hysteresis of this material show the presence of a 'curvature' shape which indicates ferromagnetic (FM) nature and a 'straight' shape which correspond to antiferromagnetic (AFM) properties. Similar magnetic hysteresis plot was reported for $\mathrm{CoO}$ nanoparticles with size ranging from 10-80 nm prepared by sol-gel method [53]. Existence of $\mathrm{CoO}$ evidenced from FTIR and XRD results (Figs. 1 and 4 ) may lead to the antiferromagnetic attributes of RH-20Co. Co nanoparticles prepared in this work is most likely following the core-shell model, in which the core is attributed to metallic Co (ferromagnetic) and the shell consists of $\mathrm{CoO}$ spesies (antiferomagnetic) [54] as confirmed by TEM images in Fig. (9). The interfacial coupling between the core and shell

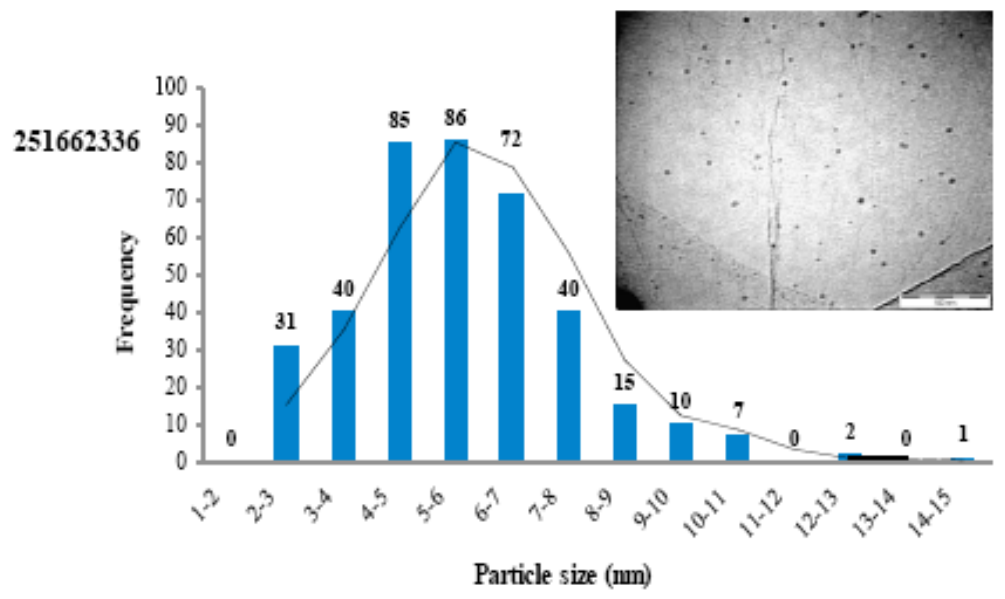

Fig. (6). Particle size distribution of the synthesized cobalt nanoparticles. Inset representative TEM image showing particles with average size $5.6 \mathrm{~nm}$.

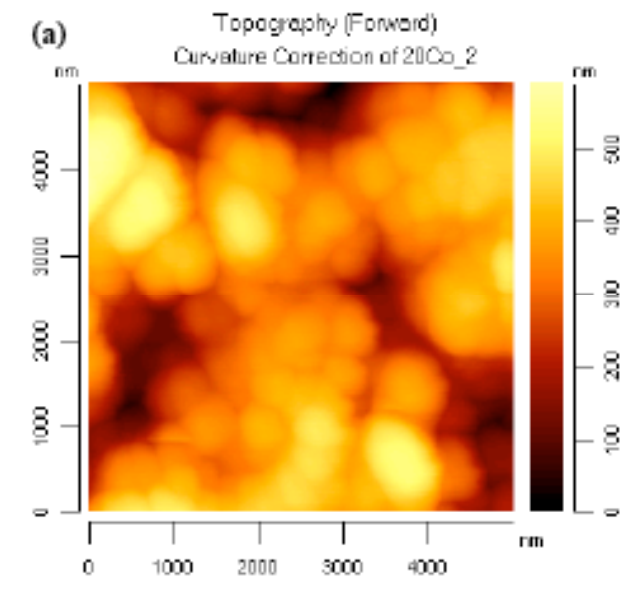

(b)
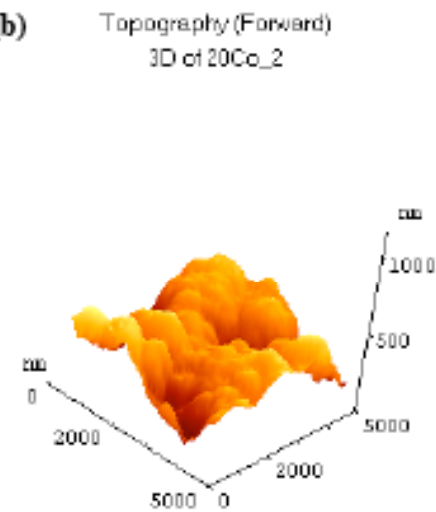

Fig. (7). The AFM images of RH-20Co. 
system can lead to higher $H_{c}$ and $M_{r}$ and results in a lower $M_{s}[55]$ as obtained in this study. However, the extent of the antiferromagnetic influence by residual Co phase is still under investigation.

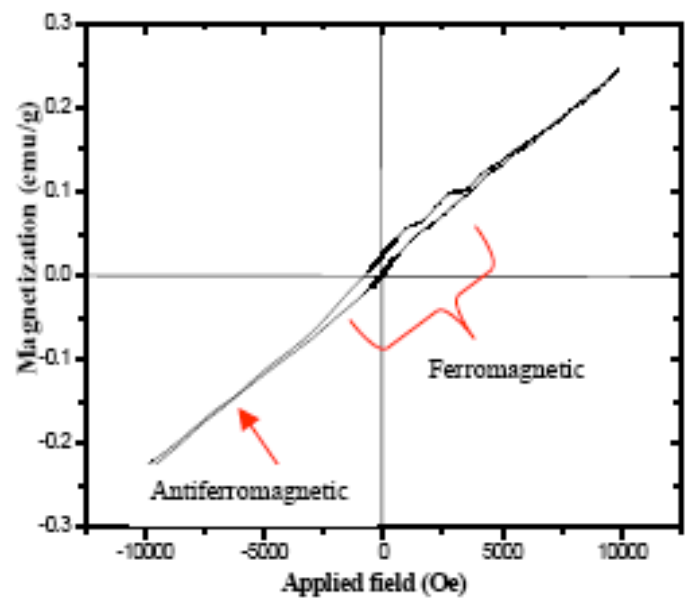

Fig. (8). Magnetization $(M)$ versus applied filed $(H)$ curve of RH$20 \mathrm{Co}$ at $300 \mathrm{~K}$.

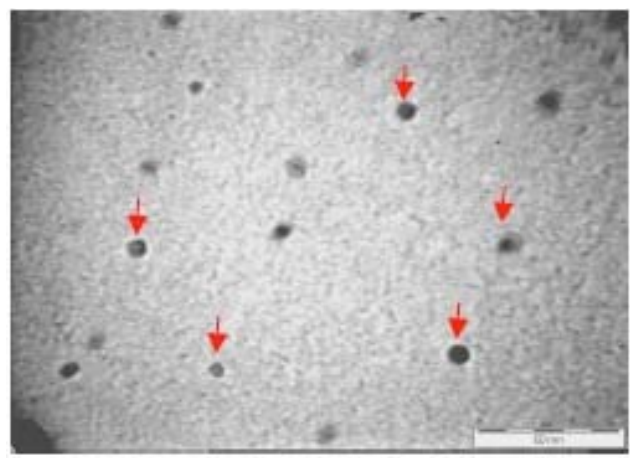

Fig. (9). TEM micrograph showing the presence of $\mathrm{CoO}$ shell for RH-20Co.

\section{CONCLUSION}

Cobalt rice husk silica nanoparticle was synthesized via a simple sol-gel method under mild conditions. This simple approach resulted in the successful generation of spherical and uniform cobalt nanoparticles ranging 2-15 nm. High BET surface area of RH-20Co suggests a good dispersion of cobalt in the siliceous matrix. The characterization by FTIR and XRD reveals the presence of $\mathrm{CoO}, \mathrm{Co}_{3} \mathrm{O}_{4}$ and $\mathrm{Co}$ silicate hydroxide in the material. The RH-20Co was found to exhibit both ferromagnetic and antiferromagnetic properties due to the core-shell system. Smaller size of the synthesized particles results in lower $M_{s}$ value in comparison to $M_{s}$ of bulk cobalt.

\section{ACKNOWLEDGEMENTS}

The authors thank Universiti Sains Malaysia for providing financial assistance through PRGS grant (1001/PKIMIA/ 842037), the E-Science fund (305/PKIMIA/613317) and the RU grant (1001/PKIMIA/814019) which partially supported this work.

\section{REFERENCES}

[1] Fleaca, C.T.; Morjan, I.; Alexandrescu, R.; Dumitrache, F.; Soare, I.; Gavrila-Florescu F. Le Normand, L.; Derory, A. Magnetic properties of core-shell catalyst nanoparticles for carbon nanotube growth. Appl. Surf. Sci., 2009, 255, 5386-5390.

[2] Cheng, G.; Walker, A.R.H. Synthesis and characterization of cobalt/gold bimetallic nanoparticles. J. Magn. Magn. Mater., 2007, 311,31-35.

[3] Tong, J.; Bo, L.; Li, Z.; Lei, Z.; Xia, C. Magnetic $\mathrm{CoFe}_{2} \mathrm{O}_{4}$ nanocrystal: A novel and efficient heterogeneous catalyst for aerobic oxidation of cyclohexane. J. Mol. Catal. A: Chem., 2009, 307, 5863 .

[4] Kalyan Kamal, S.S.; Sahoo, P.K.; Premkumar, M.; Rama Rao, N.V.; Jagadeesh Kumar, T.; Sreedhar, B.; Singh, A.K.; Ram, S.; Chandra Sekhar, K. Synthesis of cobalt nanoparticles by a modified polyol process using cobalt hydrazine complex. J. Alloys Compd., 2009, 474, 214-218.

[5] Deng, Y.-H.; Wang, C.-C.; Hua, J.-H.; Yang, W.-L.; Fu, S.-K. Investigation of formation of silica-coated magnetite nanoparticles via sol-gel approach. Colloid Surf. A., 2005, 262, 87-93.

[6] Ribeiro, R.U.; Liberatori, J.W.C.; Winnishofer, H.; Bueno, J.M.C.; Zanchet, D. Colloidal Co nanoparticles supported on $\mathrm{SiO}_{2}$ : Synthesis, characterization and catalytic properties for steam reforming of ethanol. Appl. Catal. B., 2009, 91, 670-678.

[7] Zhou, L.; Xu, J.; Miao, H.; Wang, F.; Li, X. Catalytic oxidation of cyclohexane to cyclohexanol and cyclohexanone over $\mathrm{Co}_{3} \mathrm{O}_{4}$ nanocrystals with molecular oxygen. Appl. Catal. A., 2005, 292, 223-228.

[8] Ren, L.; Wang, P.; Han, Y.; Hu, C.; Wei, B. Synthesis of $\mathrm{CoC}_{2} \mathrm{O}_{4} \cdot 2 \mathrm{H}_{2} \mathrm{O}$ nanorods and their thermal decomposition to $\mathrm{Co}_{3} \mathrm{O}_{4}$ nanoparticles. Chem. Phys. Lett., 2009, 476, 78-83.

[9] Salavati-Niasari, M.; Khansari, A.; Davar, F. Synthesis and characterization of cobalt oxide nanoparticles by thermal treatment process. Inorg. Chim. Acta, 2009, 362, 4937-4942.

[10] Yang, H.T.; Shen, C.M.; Wang, Y.G.; Su, Y.K.; Yang, T.Z.; Gao, H.J. Stable cobalt nanoparticles passivated with oleic acid and triphenylphospine. Nanotechnology, 2004, 15, 70-74.

[11] Robinson, I.; Volk, M.; Tung, L.D.; Caruntu, G.; Kay, N.; Thanh, N.T.K. Synthesis of Co nanoparticles by pulsed laser irradiation of cobalt carbonyl in organic solution. J. Phys. Chem. C., 2009, 113, 9497-9501.

[12] Wang, Z.H.; Choi, C.J.; Kim, J.C.; Kim, B.K.; Zhang, Z.D. Characterization of $\mathrm{Fe}-\mathrm{Co}$ alloyed nanoparticles synthesized by chemical vapour condensation. Mater. Lett., 2003, 57, 3560-3564.

[13] Sharma, P.K.; Dutta, R.K.; Pandey, A.C. Alteration of magnetic and optical properties of ultrafine dilute magnetic semiconductor $\mathrm{ZnO}: \mathrm{Co}^{2+}$ nanoparticles. J. Colloid Interface Sci., 2010, 345, 149153.

[14] Martinez, A.; Prieto, G. The key role of support surface tuning during the preparation of catalysts from reverse micellarsynthesized metal nanoparticles. Catal. Commun., 2007, 8, 1479 1486.

[15] Zhao, Y.-W.; Zheng, R.K.; Zhang, X.X.; Xiao, J.Q. A simple method to prepare uniform Co nanoparticles. IEEE Trans. Magn., 2003, 39, 2764-2766.

[16] Chitu, L.; Chuskin, Y.; Luby, S.; Majkova, E.; Satka, A.; Ivan, J. Smrcok, L.; Buchal, A.; Giersig, M.; Hilgendorff, M. Structure and self-assembling of Co nanoparticles. Mater. Sci. Eng. C., 2007, 27, 23-28.

[17] Haeiwa, T.; Segawa, K.; Konishi, K. Magnetic properties of isolated $\mathrm{Co}$ nanoparticles in $\mathrm{SiO}_{2}$ capsule prepared with reversed micelle. J. Magn. Magn. Mater., 2007, 310, e809-e811.

[18] Fu, W.; Yang, H.; Hari-Bala, Liu, S.; Li, M.; Zou, G. Preparation and characteristics of core-shell structure cobalt/silica nanoparticles. Mater. Chem. Phys., 2006, 100, 246-250.

[19] Mekhemer, G.A.H.; Abd-Allah, H.M.M.; Mansour, S.A.A. Surface characterization of silica-supported cobalt oxide catalysts. Colloid Surf. A., 1999, 160, 251-259.

[20] Wang, R.; Gao, B.; Jiao, W. A novel method for immobilization of Co tetraphenylporphytins on $\mathrm{P}(4 \mathrm{VP}-\mathrm{co}-\mathrm{St}) / \mathrm{SiO}_{2}$ : Efficient catalysts for aerobic oxidation of ethylbenzenes. Appl. Surf. Sci., 2009, 255, 4109-4113.

[21] Liou, T.-H. Preparation and characterization of nano-structured silica from rice husk. Mater. Sci. Eng. A., 2004, 364, 313-323. 
[22] Adam, F.; Andas, J. Amino benzoic acid modified silica-An improved catalyst for the mono-substituted product in the benzylation of toluene with benzyl chloride. J. Colloid Interface Sci., 2007, 311, 135-143.

[23] Tsay, M.-T.; Chang, F.-W. Characterization of rice husk ashsupported nickel catalysts prepared by ion exchange. Appl. Catal. A., 2000, 203, 15-22.

[24] Adam, F.; Retnam, P.; Iqbal, A. The complete conversion of cyclohexane into cyclohexanol and cyclohexanone into a simple-silica chromium heterogeneous catalyst. Appl. Catal. A., 2009, 357, 93-99.

[25] Adam, F.; Ahmed, A.E.; Sia, L.M. Silver modified porous silica from rice husk and its catalytic activity potential. J. Porous Mater., 2008, 15, 434-444.

[26] Ferreira, R.V.; Pereira, I.L.S.; Cavalcante L.C.D.; Gamarra, L.F.; Carneiro, S.M.; Amaro Jr, E.; Fabris, J.D.; Domingues, R.Z.; Andrade, A.L. Synthesis and characterization of silica-coated nanoparticles of magnetite. Hyperfine Interact., 2010, 195, 265-274.

[27] Piwon'ski, I. Mesoscopic objects, porous layers and nanocomposites-Possibilities of sol-gel chemistry. Appl. Surf. Sci., 2009, 256S, S86-S91.

[28] Sales, L.S.; Robles-Dutenhefner, P.A.; Nunes, D.L.; Mohallem, N.D.S.; Gusevskaya, E.V.; Sousa, E.M.B. Characterization and catalytic activity studies of sol-gel $\mathrm{Co}-\mathrm{SiO}_{2}$ nanocomposites. $\mathrm{Ma}$ ter. Character., 2003, 50, 95-99.

[29] Menezes, W.G.; Camargo, P.H.C.; Oliveira, M.M.; Evans, D.J.; Soares, J.F.; Zarbin, A.J.G. Sol-gel processing of a bimetallic alkoxide precursor confined in a porous glass matrix: A route to novel glass/metal oxide nanocomposites. J. Colloid Interface Sci., 2006, 299, 291-296.

[30] Zaky, R.R.; Hessien, M.M.; El-Midany, A.A.; Khedr, M.H.; AbdelAal, E.A.; El-Barawy, K.A. Preparation of silica nanoparticles from semi-burned rice straw ash. Powder Technol., 2008, 185, 31-35.

[31] Al-Oweini, R.; El-Rassy, H. Synthesis and characterization by FTIR spectroscopy of silica aerogels prepared using several $\mathrm{Si}(\mathrm{OR})_{4}$ and $\mathrm{R}$ "Si(OR') $)_{3}$ precursors. J. Mol. Struct., 2009, 919, 140-145.

[32] Huang, X.-H.; Chen, Z.-H. Sol-gel preparation and characterization of $\mathrm{CoFe}_{2} \mathrm{O}_{4}-\mathrm{SiO}_{2}$ nanocomposites. Solid State Commun., 2004, 132, 845-850.

[33] Ghattas, M.S. Cobalt-modified mesoporous FSM-16 silica: Characterization and catalytic study. Microporous Mesoporous Mater., 2006, 97, 107-113.

[34] Kababji, A.H.; Joseph, B.; Wolan, J.T. Silica-supported cobalt catalysts for Fischer-Tropsch synthesis: Effects of calcination temperature and support surface area on cobalt silicate formation. Catal. Lett., 2009, 130, 72-78.

[35] Bae, J.W.; Kim, S.-M.; Kang, S.-H.; Chary, K.V.R.; Lee, Y.-J.; Kim, H.-J.; Jun, K.-W. Effect of support and cobalt precursors on the activity of Co/AlPO4 catalysts in Fischer Tropsch synthesis. $J$. Mol. Catal. A: Chem., 2009, 311, 7-16.

[36] Salavati-Niasari, M.; Fereshteh, Z.; Davar, F. Synthesis of cobalt nanoparticles from [bis(2-hyroxyacetophenato)cobalt(II)] by thermal decomposition. Polyhedron, 2009, 28, 1065-1068.

[37] Coulter, K.E.; Sault, A.G. Effects of activation on the surface properties of silica-supported cobalt catalysts. J. Catal., 1995, 154, 5664.
[38] Carvalho, W.A.; Wallau, M.; Schuchardt, U. Iron and copper immobilised mesoporous MCM-41 molecular sieves as catalysts for the oxidation of cyclohexane. J. Mol. Catal. A: Chem., 1999, 144, 91-99.

[39] El Haskouri, J.; Cabrera, S.; Gomez-Garcia, C.J. High cobalt content mesoporous silica. Chem. Mater., 2004, 16, 2805-2813.

[40] Bhoware, S.S.; Singh, A.P. Characterization and catalytic activity of cobalt containing MCM-41 prepared by direct hydrothermal, grafting and immobilization methods. J. Mol. Catal. A: Chem., 2007, 266, 118-130.

[41] Shi, G.; Shen, J. Skeletel isomerisation of 1-hexene over sulfided Co/Co-MCM-41 catalysts. Energy Fuels, 2009, 23, 320-326.

[42] Li, J.; Xn, X.; Hao, Z.; Zhao, W. Mesoporous silica supported cobalt oxide catalysts for catalytic removal of benzene. J. Porous Mater., 2008, 15,163-169.

[43] Tang, L.; Li, B.; Zhai, Z.; Li, J.; Ou, E.; Wang, J. Selective oxidation of diphenylmethane over cobalt-doped mesoporous titaniasilica catalyst with high Ti content. Catal. Lett., 2008, 121, 63-69.

[44] Ojeda, M.; Perez-Alonso, F.J.; Terreros, P.; Rojas, S.; Herranz, T.; Granados, M.L.; Fierro, J.L.G. Silylation of a $\mathrm{Co} / \mathrm{SiO}_{2}$ catalyst. Characterization and exploitation of the $\mathrm{CO}$ hydrogenation reaction. Langmuir, 2006, 22, 3131-3137.

[45] Lim, W.T.L.; Zhong, Z.; Borgna, A. An effective sonicationassisted reduction approach to synthesize highly dispersed Co nanoparticles on $\mathrm{SiO}_{2}$. Chem. Phys. Lett., 2009, 471,122-127.

[46] Dutta, P.; Dunn, B.C.; Eyring, E.M.; Shah, N.; Huffman, G.P.; Manivannan, A.; Seehra, M.S. Characteristics of cobalt nanoneedles in $10 \%$ Co aerogel Fischer-Tropsch catalyst. Chem. Mater., 2005, 17, 5183-5186.

[47] Jalama, K.; Coville, N.J.; Hildebrandt, D.; Glasser, D.; Jewell, L.L. Effect of cobalt carboxylate precursor chain length on FisherTropsch cobalt/alumina catalysts. Appl. Catal. A., 2007, 326, 164172 .

[48] Jablonski, J.M.; Wolcyrrz, M.; Krajczyk, L. On cobalt silicate formation during high-temperature calcinations of impregnated cobalt/silica catalysts. J. Catal., 1998, 173, 530-534.

[49] Bi, H.; Jiang, X.; Yang, C.; Hong, J. Synthesis of cobalt disulfide nanoparticles in polymer matrix. Mater. Lett., 2003, 57, 2606-2611.

[50] Ming, H.; Baker, B.G. Characterization of cobalt Fischer-Tropsch catalysts I. Unpromoted cobalt-silica gel catalysts. Appl. Catal. A., 1995, 123, 23-26.

[51] den Breejen, J.P.; Sietsma, J.R.A.; Friedrich, H.; Bitter, J.H.; de Jong, K.P. Design of supported cobalt catalysts with maximum activity for the Fischer-Tropsch synthesis. J. Catal., 2010, 270,146-152.

[52] Klabunde, K.J. Nanoscale Materials in Chemistry; Wiley Interscience: New York, 2001, p. 198.

[53] Zhang, L.; Xue, D.; Guo, C. Anomalous magnetic properties of antiferromagnetic $\mathrm{CoO}$ nanoparticles. J. Magn. Magn. Mater., 2003, 267, 111-114.

[54] Hong, J.S.; Pyun, J.; Park, Y.W.; Kim, C.S.; Shin, In-Bo. Oxidation effect in cobalt nanoparticles magnetic fields. IEEE Trans. Magn., 2009, 45, 2464-2466.

[55] Pirkkalainen, K.; Leppanen, K.; Vainio, U.; Webb, M.A.; Elbra, T.; Kohout, T.; Nyk"anen, A.; Ruokolainen, J.; Kotelnikova, N.; Serimaa, R. Nanocomposites of magnetic cobalt nanoparticles and cellulose. Eur. Phys. J. D., 2008, 49, 333-342.

This is an open access article licensed under the terms of the Creative Commons Attribution Non-Commercial License (http://creativecommons.org/licenses/by-nc/3.0/) which permits unrestricted, non-commercial use, distribution and reproduction in any medium, provided the work is properly cited. 